\title{
The rapid plasma reagin (circle) card test in biological false positive and leprosy sera
}

\author{
M. F. GARNER AND J. L. BACKHOUSE \\ From the Institute of Clinical Pathology and Medical Research, Lidcombe, New South Wales, Australia
}

SYNOPSIS The rapid plasma reagin (RPR) circle card and the Venereal Disease Reference Laboratory (VDRL) slide test results were compared on a group of sera known to show biological false positive (BFP) reactions to reagin detection tests for syphilis. The RPR test was more specific than the N VDRL test on the sera selected, ie, it gave fewer false positive results than the VDRL test. However, in a group of presumed normal sera, the RPR test gave BFP reactions while the VDRL test gave none.

The RPR test gave fewer BFP reactions than the VDRL test in 269 sera from patients with lepromatous leprosy.

A biological false positive (BFP) reaction is a reactive result (to include both strong and weak quantitative results) to a reagin detection test and a non-reactive result to a treponemal test in the serum of a person who has no history or clinical signs of treponemal infection. The cardiolipin Wassermann reaction (CWR), Veneral Disease Research Laboratory (VDRL) test, and the rapid plasma reagin (RPR) circle card test are all reagin detection tests. The Treponema pallidum immobilization (TPI) and fluorescent treponemal antibody absorption (FTAABS) tests are specific tests for treponemal infection.

Reagin is present in all human serum in amounts not usually detectable by the CWR and VDRL test. An increase in reagin in the serum can be caused by a wide variety of conditions, eg, treponemal infection (syphilis and yaws), leprosy, pregnancy, recent vaccination, collagen diseases, respiratory infections, etc. Clinical signs, history, and the results of the TPI and FTA-ABS tests separate those reactive reagin detection tests due to treponemal infection from those which are BFP reactions. It must be remembered that syphilis or yaws can occur in people who have leprosy or are pregnant, ie, who have conditions known to cause BFP reactions.

One of the most commonly used screening tests for syphilis today is the VDRL slide test. This test is carried out using serum which has been heated at $56^{\circ} \mathrm{C}$ for 30 minutes. The serum is placed in a circle on a slide and prepared antigen suspension added. The slide is then rotated at $180 \mathrm{rpm}$ for four Received for publication 8 June 1972. minutes and the test results are read microscopically. The RPR circle card test is performed using either plasma or unheated serum. The plasma or serum is placed in a circle on a special card, antigen addee and the card rotated at $100 \mathrm{rpm}$ for eight minutes The results are read macroscopically.

The RPR circle card test is available in a disposable kit form from Hynson, Westcott, and Dunning Inc, Baltimore, USA. Each kit contains everything required for the test-droppers, cards, antigen, etc. The antigen is used as supplied in the kit; no preparation is necessary. Rapid plasma reagin antigen is VDRL antigen to which choline chloride EDTA, and charcoal particles have been added.

In recent surveys the RPR card test has been shown to be more sensitive but less specific than the VDRL test (Walker, 1971; Garner and Backhouse, 1972).

This survey was carried out to compare the results of the RPR circle card test with those of the VDRL slide test on, first, a group of sera which were considered to show BFP reactions, and secondly on sera from patients who had lepromatous leprosy.

\section{Materials and Methods}

Two hundred and three sera showing BFP reactions were tested using the disposable RPR circle card test kits and the technique describad by Portnoy (1963). These BFP sera had all shown reactive CWR and/or VDRL test results and non-reactive TPI and FTAABS test results. 
Sera from 269 patients with lepromatous leprosy were tested using the CWR, RPR, VDRL, TPI, and FTA-ABS tests.

A control group of 420 presumed normal sera were also tested. These sera were non-reactive in the CWR, VDRL, TPI, and FTA-ABS tests.

\section{Results}

\section{BIOLOGICAL FALSE POSITIVE SERA}

In the total group of 203 sera, the RPR test was reactive in 156, the VDRL test in 189, and the CWR in 58. The RPR and VDRL tests were both reactive in 149 sera. In seven sera the CWR and RPR tests were reactive and the VDRL test not reactive. There were $\mathbf{4 0}$ sera in which the RPR test was not reactive and the VDRL test or CWR and VDRL tests were reactive. Seven sera were reactive in the CWR only (Table I).

\begin{tabular}{|c|c|c|c|c|c|c|c|}
\hline & \multirow[b]{2}{*}{$\begin{array}{l}\text { Total } \\
\text { Sera }\end{array}$} & \multicolumn{6}{|c|}{ Reactive Test Results } \\
\hline & & $\begin{array}{l}C W R \\
\text { Only }\end{array}$ & $\begin{array}{l}\text { VDRL } \\
\text { Only }\end{array}$ & $\begin{array}{l}C W R \\
\& \\
V D R L\end{array}$ & $\begin{array}{l}C W R \\
\& \\
L R P R\end{array}$ & $\begin{array}{l}R P R \\
\& \\
V D R L\end{array}$ & $\begin{array}{l}C W R, \\
R P R, \\
L \& \\
V D R L\end{array}$ \\
\hline Antenatal & 67 & 1 & 13 & 1 & 3 & 35 & 14 \\
\hline Blood donors & 55 & 1 & 1 & - & 1 & 44 & 8 \\
\hline Recent vaccination & 9 & - & 2 & 1 & - & 4 & 2 \\
\hline Collagen disease & 4 & - & - & - & - & 2 & 2 \\
\hline No history & 42 & 2 & 10 & 1 & 3 & 16 & 10 \\
\hline Miscellaneous & 26 & 3 & 10 & 1 & - & 8 & 4 \\
\hline Total BFP sera & 203 & 7 & 36 & 4 & 7 & 109 & 40 \\
\hline
\end{tabular}

Table I Results of CWR, RPR, and VDRL tests on 203 BFP sera

The BFP sera were divided into six groups. These were sera from antenatal patients (67), blood donors (55), recently vaccinated people (9), patients with collagen disease (4), people about whom no history was obtainable (42), and a miscellaneous group (26) of people with a wide variety of illnesses. The results of the RPR and VDRL tests in these six groups are shown in Table $I$. In all these groups the number of sera reactive in the VDRL test equalled or exceeded the number reactive in the RPR test.

\section{LEPROSY SERA}

There were 269 sera from patients with lepromatous leprosy in this group. The TPI and FTA-ABS tests were reactive in 14 of these sera, indicating that they came from patients who had both syphilis and leprosy. The RPR and VDRL tests were both reactive in 11 of these sera, the RPR test only in two and the VDRL test only in one (Table II).

Sera from the remaining 255 patients were not reactive in the TPI and FTA-ABS tests indicating

\begin{tabular}{lllllll}
\hline $\begin{array}{l}\text { No. of } \\
\text { Sera }\end{array}$ & \multicolumn{2}{l}{ Reactive Test Results } & & & \\
\cline { 2 - 7 } & $\begin{array}{l}\text { CWR } \\
\text { Only }\end{array}$ & $\begin{array}{l}\text { VDRL } \\
\text { Only }\end{array}$ & $\begin{array}{l}\text { RPR } \\
\text { Only }\end{array}$ & $\begin{array}{l}\text { RPR \& } \\
\text { VDRL }\end{array}$ & $\begin{array}{l}\text { CWR, } \\
\text { RPR \& } \\
\text { VDRI. }\end{array}$ & $\begin{array}{l}\text { TPI \& } \\
\text { ABS }\end{array}$ \\
\hline 14 & - & 1 & 2 & 6 & 5 & 14 \\
255 & 2 & 22 & 10 & 2 & - & - \\
\hline
\end{tabular}

Table II $R P R$ and VDRL test results on 269 leprosy sera

that these people had not had syphilis. Biological false positive reactions were found in 36 of the 255 sera as follows: CWR only reactive in two sera, VDRL test only reactive in 22 sera, RPR test only reactive in 10 sera, and RPR and VDRL tests reactive in two sera. Thus the RPR test showed false positive results in 12 and the VDRL test in 24 sera from lepromatous leprosy patients (Table II). Seventeen of these sera were from females and 19 from males.

\section{PRESUMED NORMAL SERA}

The RPR circle card test was reactive in 11 of the 420 sera in this group. These reactive sera were from antenatal patients (6), blood donors (3), and a miscellaneous group (2).

\section{Discussion}

In the group of BFP sera in this survey the RPR test showed fewer false positive results than the VDRL test, indicating that, in this particular group of sera, the RPR test was more specific than the VDRL test. This could be misleading if applied generally, as the sera selected for study were those already considered to show BFP reactions on the basis of reactive CWR and/or VDRL test results and non-reactive TPI and FTA-ABS test results. It was not possible to make allowance in this selection for those sera which may show BFP reactions in the RPR test only. False positive results to the RPR test only were found in the group of normal sera tested, indicating that in these sera the RPR test was less specific than the VDRL test.

The VDRL and RPR tests both detect the antibody reagin and basically use the same antigen. The majority of BFP sera in this study have been reactive in both tests. However there are a certain number of sera which will show BFP reactions in either the RPR or the VDRL test but not in both. It would bz reasonable to expect the RPR test to show more BFP reactions than the VDRL as it is the more sensitive test (Walker, 1971), and thus presumably detects reagin more readily.

The RPR and VDRL tests were equally sensitive in detecting the small number of leprosy patients who 
had syphilis. In those leprosy patients who had no serological or clinical evidence of syphilis, the RPR test was more specific than the VDRL test, ie, it gave fewer false positive results. Portnoy, Brewer, and Harris in 1962 found the RPR test more specific than the VDRL test in leprosy sera. They tested 27 sera and found the RPR test non-reactive in all 27, whereas the VDRL test was reactive in five. In the present series both tests gave BFP reactions in sera from patients with lepromatous leprosy. The VDRL test showed twice the number of false positive results of the RPR test. Both tests were reactive in only two of the 36 sera showing BFP reactions. It appears from the results in this survey that in sera from patients with lepromatous leprosy the RPR test may be more satisfactory than the VDRL test as a screening test for treponemal infection.

The main use of the VDRL and RPR tests is as screening tests for syphilis. In this role sensitivity is more important than specificity, as reactive screening test results can be confirmed by using the specific TPI and FTA-ABS tests. In this way BFP reactions can be distinguished from those due to syphilis or $\vec{F}$ other treponemal infection. However, unless there is some history or clinical indication for specific tests, a false negative test result is accepted as a true negative and the patient is not investigated further.

References

Garner, M. F., and Backhouse, J. L. (1972). The rapid plasma reagin $\vec{\circ}$ (circle) card test. A screening test for syphilis. Med.J. Aust., in press.

Portnoy, J., Brewer, J. H., and Harris, A. (1962). Rapid Plasma Reagin Card Test for Syphilis and Other Treponematoses. Publ. Hlth Rep., 77, 645-652.

Portnoy, J. (1963). Modifications of the rapid plasma reagin (RPR) card test for syphilis, for use in large scale testing. Amer.J. clin. G Path., 40, 473-479.

Walker, A. N. (1971). Rapid plasma reagin (RPR) card test. A screening method for treponemal disease. Brit. J. vener. Dis., 47, 259-262. 